\title{
Chronic facial pain: a multidisciplinary problem
}

\author{
G Madland, C Feinmann
}

\begin{abstract}
Atypical facial pain is an unrecognised and unhelpful diagnosis but one which describes chronic pains that do not fit the present classification system. Due to the site of the pain, patients may seek and, indeed, receive treatment from dental practitioners and specialists, but the pain is often unresponsive and may have more in common with unexplained medical symptoms affecting other areas of the body, than with other dental symptoms. This review suggests a need for a diagnostic category of "chronic facial pain", which demands a multidisciplinary approach to diagnosis and management. (F Neurol Neurosurg Psychiatry 2001;71:716-719)
\end{abstract}

Keywords: chronic pain; facial pain

Chronic idiopathic orofacial pain is an ill understood group of conditions, which may involve the whole of the mouth and face. Unfortunately descriptions of disorders and treatment tend to be influenced by the background of the specialist assessing the patient. Thus patients who see maxillofacial surgeons have symptoms described in terms of clicking, sticking, and locking of the temporomandibular joint and pain in the associated musculature. Ear, nose, and throat surgeons may retain Costen's outdated notion that the pain is due to missing molar teeth and may refer on to maxillofacial surgeons or restorative dental specialists. Despite advice from the National Institute of Health that "there is no evidence linking occlusal abnormalities with pain", patients' occlusions continue to be adjusted by ill informed practitioners, often leading to more problems for patients.

The NIH conference in 1996 reviewed the issues on the management of orofacial pain, concluding that major problems hampered present diagnostic classifications and treatment. Five years later, there is no greater clarity in classification.

Current diagnoses include tension headache, migraine, neckache, temporomandibular disorder (temporomandibular joint pain dysfunction syndrome, facial arthromyalgia), and atypical facial pain. These pains seem to arise from blood vessels, muscles, and joint capsules rather than conforming to the distribution of sensory nerve branches, as in trigeminal neuralgia. Artificial distinctions in clinical presentation lead patients to different specialists providing different treatments, including dentists, neurologists, otorhinologists, osteopaths, chiroporacters, and psychiatrists, with little collaboration.

There are additional important problems concerning the recognition and definition of underlying psychiatric disturbances. Emotional disturbance, when present, is often mild and of brief duration and psychiatric classification has proved an inadequate measure.

\section{Chronic symptoms}

Chronic symptoms and syndromes pose a major challenge to medicine: they are common, often persistent, and are associated with significant distress, disability, and unnecessary expenditure of medical resources. In United Kingdom primary care, somatic symptoms and syndromes account for $20 \%$ of consultations. Among medical outpatients, somatic complaints accounted for 35\% of new referrals in a United Kingdom study. ${ }^{2}$ Even among medical inpatients, a substantial proportion have complaints that are found to be functional. The prevalence of emotional distress and disorder in patients who attend hospital with unexplained syndromes (such as irritable bowel syndrome) is higher than in patients with comparable medical conditions (such as inflammatory bowel disease) and many such patients are severely disabled. ${ }^{3}$

Wessely et $a l^{\beta}$ suggest that each medical specialty has defined its own syndrome or syndromes in terms of symptoms that relate to their organ or interest, despite similarities in case definition, reported symptoms, sex, outlook, and response to treatment. Patients seek help from doctors for symptoms, and doctors diagnose diseases to explain them. Symptoms are the patient's subjective experience of changes in his or her body, diseases are objectively observable abnormalities in the body. Difficulties arise when the doctor can find no objective changes to explain the patient's subjective experience. The symptoms are then referred to as medically unexplained or functional. Atypical facial pain is relegated to the dental rather than neurological domain. Wessely et al postulate that "the existence of specific somatic syndromes is largely an artefact of medical specialisation. That is to say that the differentiation of specific syndromes reflects the tendency of specialists to focus on only those symptoms pertinent to their specialty, rather than any real differences between patients" ( $p$ 936). 


\section{Symptoms and signs}

There are four recognisable symptom complexes of chronic orofacial pain, which may, however, coexist: temporomandibular disorder (myofascial face pain); atypical facial pain (atypical facial neuralgia); atypical odontalgia (phantom tooth pain); and burning mouth syndrome (oral dysaesthesia, glossodynia, glossopyrosis). They may be considered as medically unexplained symptoms affecting four regions of the mouth and face.

Atypical facial pain is no longer included in the International Association for the Study of Pain's "classification of chronic pain". The term originated to distinguish the condition from "typical" trigeminal neuralgia, as the pain neither follows the distribution of the peripheral nerve, nor responds to antiepileptic agents. However, the categorisation of patients with similar pain histories into a diagnostic pigeon hole labelled "atypical" is self contradictory, and the definition of a condition by what it is not, rather than what it is, is unsatisfactory. A better term might be "chronic facial pain", as the defining characteristics are longevity and site, as distinct from temporomandibular disorder which affects the jaw rather than the (mid-)face, and intraoral pains.

The pain is usually a continuous dull ache with intermittent severe episodes, primarily affecting areas of the face other than joints and muscles of mastication, such as the zygomatic maxilla. Pain may be bilateral and will often have been present for several years. Analgesics are ineffective.

Atypical odontalgia has a similar character but is localised to one or more premolar or molar teeth, simulating pulpitis. ${ }^{5}$ There may be a history of inappropriate dental treatment, including extraction, and subsequent recurrence of symptoms apparently from another tooth.

Patients often attribute their pain to an antecedent event such as a dental procedure, or minor trauma to the face. Despite the notorious unreliability of such retrospective reports, these attributions have led to the suggestion that chronic facial pain may be a deafferentation syndrome (compare phantom tooth pain). ${ }^{6}$

Similarities to postamputation pains include burning quality, description as severe despite lack of sleep disturbance, poor localisation, and delay between injury and incidence. The lack of response to nerve block is also a common feature. Divergence from a classic picture may well betray the influence of factors beyond identifiable pathology. Depression is a common feature but is more likely to be a consequence of living with pain than a precursor to it.

Ninety five per cent of patients with atypical facial pain complain of other symptoms, including headache, neck and backache, dermatitis or pruritis, irritable bowel, and dysfunctional uterine bleeding. This prevalence is much greater than in the normal population. ${ }^{8}$

Positron emission tomography has demonstrated an increased contralateral cingulate cortex activity (and decreased prefrontal cortex activity), in response to both heat and nociceptive stimuli, in patients with atypical facial pain relative to controls. ${ }^{9}$ This is suggestive of an exaggerated perception of pain in response to peripheral stimuli but how this might develop remains a matter for speculation. The authors thought it likely that the mechanism for the differences found is related to anxiety and attention and therefore that the pain might be brought under conscious control. However, the cross sectional nature of this evidence precludes any conclusions as to whether such central changes are primary or secondary to pain.

\section{Epidemiological considerations}

Although the prevalence of joint related and muscle related symptoms has been studied, the epidemiology of chronic facial pain remains unclear. An obvious explanation for why some people choose to consult whereas others do not is the severity of their symptoms. Sadly, epidemiological studies of facial pain have tended to ignore intensity and frequency of symptoms and recorded only their presence or absence.

Orofacial pain is a common problem affecting at least $10 \%$ of the adult population and $50 \%$ of the elderly population, and its inadequate recognition and management present an enormous problem to the health service. The sex distribution is thought to be equal in the general population, but four times as many women as men seek help. ${ }^{10}$ Barksy and Borus have described how changes in social attitudes have reduced the public tolerance to mild symptoms and benign infirmities, physiological responses to anxiety are also misinterpreted as illness. Isolated symptoms are then identified as disease by patients. ${ }^{11}$

\section{Associated personality and psychiatric problems}

There is a long standing association in the literature between chronic facial pain and psychological distress, particularly depression. ${ }^{12}$ This is consistent with other chronic pain groups, in which the prevalence of depressive symptoms is consistently higher than in the general population and many other medical populations. ${ }^{13}$ Studies comparing patients with organic lesions and patients with indeterminate chronic pain, report considerably higher prevalence of depression among the second. ${ }^{14}$ This suggests that beliefs and attributions about the cause of their symptoms may affect psychological wellbeing in patients with chronic pain.

A "diathesis-stress" framework has been proposed to explain the high comorbidity between chronic pain and depression. ${ }^{15}$ This approach encourages identification of vulnerability factors in the individual person as well as investigation into the nature of the stressor, and may be a useful theoretical basis from which to advance the study of depression in chronic pain.

\section{Management}

The treatment offered to a patient with chronic facial pain will be determined by the specialist understanding of the clinician to whom he or she is referred. ${ }^{16} \mathrm{~A}$ multidisciplinary approach 
is therefore preferable and should be adopted for diagnostic and prognostic assessment. Possible dental or neurological causes, including trigeminal or craniocervical neuropathy, must be eliminated with clinical and radiographic investigation by a specialist, but extensive investigation can lead to patients being off loaded from specialist to specialist in search of a diagnosis, and feeling ill understood, overinvestigated, and dissatisfied. Patients want to know what is wrong with them rather than what is not wrong. Recent evidence suggests that it may be more helpful to assess patients in terms of disability and coping strategies, rather than pain intensity itself. ${ }^{17}$ There is a real need to make diagnosis realistic in terms of what patients are told and what they understand about their problems. ${ }^{18} 19$ Patients must feel that the physician believes in their pain and that the patient's understanding or attribution of the pain is accepted. It is crucial that physicians and patients reach an agreement about how to manage the pain. Thereafter, the mainstays of treatment are counselling and antidepressant medication. There is no evidence from randomised trials of benefit from surgical intervention, which should in itself be enough to discourage such an intervention, even in the face of patient entreaty. Patients with chronic pain are often desirous of a concrete cause and treatment, and will therefore request surgery, but there is reason to think that surgery may in fact worsen the problem.

In a 4 year review of a mixed group of 71 patients with "idiopathic" facial pain who had initially participated in a double blind trial of dothiepin, and received further courses of the drug and counselling sessions, $43 \%$ of the patients with chronic facial pain were pain free. ${ }^{8}$ The "atypical" patients were the least responsive.

Pain relief with tricyclic agents seems to be independent of the antidepressant effect of the drugs. ${ }^{20-22}$ These drugs are considered to act by altering the sensory discriminative component of pain. The possibility of interference with serotonin reuptake in the brain stem has been proposed. ${ }^{22}$ In two short term studies, the serotonin antagonist iprazochrome had equivocal effects on a group of 30 patients with chronic facial pain ${ }^{23}$ but fluoxetine improved pain severity and distress in a group of 178 non-depressed patients with chronic facial pain. $^{24}$

Unfortunately, dental and medical practitioners are often reluctant to prescribe antidepressant agents, mistakenly considering them to be addictive or to have overwhelming side effects. In addition, inadequate dosage and duration, perhaps in combination with expressed lack of confidence in their efficacy, prevent the drugs from having any beneficial effect. The liaison psychiatrist is currently the only specialist appropriately trained in drug treatments.

Cognitive behavioural therapy, in combination with drug treatment, has been found to reduce the pain's interference with life and to increase perceived control over life, in patients with chronic facial pain. ${ }^{24}$ Effective communication during the early phases of facial pain may prevent the development of long term problems. Clinicians should be aware of the importance of listening to the patient's beliefs about their pain, and of trying to address their concerns without resorting to outdated psychogenic models of pain. Patients gain enormous benefit from having their symptoms acknowledged as part of a group of conditions with which clinicians are familiar. An information booklet is also likely to be helpful (available from the authors on request). Reassurance about the non-malignant nature of chronic pain is also important, but empty promises that "things will get better" are unhelpful. The message is that talking to patients is often more useful, albeit sometimes more demanding, than operating on them.

\section{Conclusions}

(1) "Chronic facial pain" is a more descriptive term than "atypical facial pain" and should be adopted for continuous, dull pain in the face, of greater than 6 months duration, with intermittent severe episodes. It differs from temporomandibular disorder and burning mouth syndrome, in terms of site (mid-face), and longevity (lacking a responsive acute formcompare temporomandibular disorder), but, once established, all have features in common with other medically unexplained conditions, and require a multidisciplinary approach.

(2) The management of this heterogeneous group of patients with pain is complicated by the area of the body involved, and by the interaction of organic and psychological factors in the somatising process. No single clinical specialty receives the training required for the differential diagnosis and management of these pain disorders and, hence, there is both a need for multidisciplinary clinics, and for specific training programmes in dental and medical undergraduate curricula.

(3) Although there is an obvious need for prompt elimination of possible organic disease such as tumour, there is also the danger of overzealous investigation and provision of inappropriate and irreversible dental treatment. General dental and medical practitioners must appreciate the risk of exacerbation of associated psychological distress and the importance of psychological assessment and counselling at an early stage.

(4) Drugs such as dothiepin and fluoxetine are of proved, if modest, benefit in the management of chronic idiopathic orofacial pain, although originally developed as antidepressant drugs. Provision of information, drug treatment, and counselling, are complemented by cognitive therapy specifically aimed at reducing interference with life.

1 National Institute of Health: technology assessment statement. Management of temporomandibular disorders. Washington, DC: NIH, 1996

2 Hamilton J, Campos R, Creed F. Anxiety, depression and management of medically unexplained symptoms in medimanagement of medically unexplained symptoms in

3 Wessely S, Nimnuan C, Sharpe M. Functional somatic symptoms: one or many? Lancet 1999;354:936-9.

4 Merskey H, Bogduk N. Classification of chronic pain. 2 nd ed. Seattle: IASP Press, 1994:59-60. 
5 Graff-Radford S, Solberg W. Atypical odontalgia. Fournal of Craniomandibular Disorders and Oral Facial Pain 1992;6: Cranionan.

6 Marbach JJ. Is phantom tooth pain a deafferentation (neuropathic) syndrome? Part I: evidence derived from pathophysiology and treatment. Oral Surgery Oral Medicine and Oral Pathology 1993;75:95-105.

7 Dohrenwend BP, Raphael KG, Marbach JJ, et al. Why is depression comorbid with chronic myofascial face pain? A family study test of alternative hypotheses. Pain 1999;83: 183-92.

8 Feinmann C. The long-term outcome of facial pain treatment. F Psychosom Res 1993;37:381-7.

9 Derbyshire SW, Jones AK, Devani P, et al. Cerebral responses to pain in patients with atypical facial pain measured by positron emission tomography. 7 Neurol Neurosurg Psychiatry 1994;57:1166-72.

10 Feinmann C. Idiopathic orofacial pain: a multidisciplinary problem. Pain: an updated review. Seattle: IASP Press, 1996 problem.

11 Barsky AJ, Borus JF. Somatization and medicalization in the era of managed care. FAMA 1995;274:1931-4

12 Mock D, Frydman W, Gordon A. Atypical facial pain: a retrospective study. Oral Surgery Oral Medicine Oral Pathology 1985;59:472-4.

13 Romano J, Turner J. Chronic pain and depression: does the evidence support a relationship? Psychol Bull 1985;97:18-34.

14 Magni G. On the relationship between chronic pain and depression when there is no organic lesion. Pain 1987;31 $1-21$.

15 Banks S, Kerns R. Explaining high rates of depression in chronic pain: a diathesis-stress framework. Psychol Bull 1996;119:95-110.
16 Raphael K, Marbach JJ. Evidence-based care of musculoskeletal facial pain: implications for the clinical science of dentistry. F Am Dent Assoc 1997;128:73-9.

17 Madland G, Feinmann C, Newman S. Factors associated with anxiety and depression facial arthromyalgia. Pain 2000;84:225-32.

18 Donovan JL, Black DR. Qualitative study of interpretation of reassurance among patients attending rheumatology of reassurance among patients attending rheumatology 541-4.

19 Clark NM, Gong M. Management of chronic disease by practitioners and patients: are we teaching the wrong things? BMF 2000;320:572-5.

20 Feinmann C, Harris M. Psychogenic facial pain. Part 2: management and prognosis. Br Dent f 1984:156:205-12.

21 Sharav Y, Singer E, Schmidt E, et al. The analgesic effect of amitriptyline on chronic facial pain. Pain 1987;31:199209.

22 Pettengill C, Reisner-Keller L. The use of tricyclic antidepressants for the control of chronic orofacial pain. fournal of Craniomandibular Practice 1997;15:53-6.

23 Hampf G. Effect of serotonin antagonists on patients with atypical facial pain. Fournal of Craniomandibular Disordorders and Facial Oral Pain 1989;3:211-12.

24 Harrison S, Glover L, Maslin L, et al. A comparison of antidepressant medication alone and in conjunction with antidepressant medication alone and in conjunction with pain. In: Jensen T, Turner J, Weisenfeld-Hallin Z, eds. Proceedings of the 8th World Congress on Pain: progress in pain research and management. Vol 8. Seattle: IASP Press, 1997.

\title{
7th European Forum on Quality Improvement in Health Care
}

\author{
21-23 March 2002 \\ Edinburgh, Scotland
}

We are delighted to announce this forthcoming conference in Edinburgh. Authors are invited to submit papers (call for papers closes on Friday 5 October 2001) and delegate enquiries are welcome.

The themes of the Forum are:

- Leadership, culture change, and change management

- Achieving radical improvement by redesigning care

- Health policy for lasting improvement in health care systems

- Patient safety

- Measurement for improvement, learning, and accountability

- Partnership with patients

- Professional quality: the foundation for improvement

- Continuous improvement in education and training

- People and improvement.

Presented to you by the BMJ Publishing Group (London, UK) and Institute for Healthcare Improvement (Boston, USA). For more information contact: quality@bma.org.uk or look at the website www.quality.bmjpg.com. Tel: +44 (0)20 7383 6409; fax: +44 (0)20 73736869 . 